\title{
ERRATUM
}

Takahide Kato - Yoshinori Matsumura

Atsuko Tsukanaka - Takeshi Harada

Mitsuo Kosaka · Nobuo Matsui

\section{Effect of low oxygen inhalation on changes in blood pH, lactate, and ammonia due to exercise}

Published online: 31 March 2004

(C) Springer-Verlag 2004

\section{Eur J Appl Physiol (2004) 91:296-302}

In Table 1, the values were not correctly aligned. The correct version is given below.

Table 1 Exercise,

cardiorespiratory and blood gas parameters influenced by exhaustive work at normoxia $(M A X)$ and at hypoxia (HYPOXIA), as well as at the workload imposed during hypoxia but carried out at normoxia $(S U B M A X)$ in six subjects. Mean values with standard deviations

are parameters determined in the state of HYPOXIA, which differ significantly $(P<0.05)$ :

from the state of MAX

${ }^{\S}$ from the state of SUBMAX

\begin{tabular}{|c|c|c|c|c|}
\hline & & MAX & HYPOXIA & SUBMAX \\
\hline \multicolumn{2}{|l|}{ Performance time (min) } & $14 " 48(2 ” 10)$ & $11 ” 01(0 " 59)^{*}$ & $11 ” 01(0 " 59)^{*}$ \\
\hline \multicolumn{2}{|l|}{ Maximal workload (watt) } & $290(45)$ & $213(33)^{*}$ & $213(33)^{*}$ \\
\hline \multicolumn{2}{|l|}{$V \mathrm{O}_{2} \max \left(\mathrm{ml} \cdot \mathrm{kg}^{-1} \cdot \min ^{-1}\right)$} & $45.25(2.12)$ & $36.73(5.03)^{*}$ & $39.12(3.17)^{*}$ \\
\hline \multirow[t]{3}{*}{ Heart rate (beats $\cdot \mathrm{min}^{-1}$ ) } & Pre-exercise & $71.7(4.9)$ & $82.5(9.7)^{* 8}$ & $73.0(6.7)$ \\
\hline & Exhaustion & $195.3(4.7)$ & $188.2(3.0)^{* \S}$ & $174.5(7.4)^{*}$ \\
\hline & At 30 min post-exercise & $102.2(9.2)$ & $97.5(10.6)$ & $82.7(18.2)^{*}$ \\
\hline \multirow[t]{3}{*}{$V_{\mathrm{E}}\left(1 \cdot \mathrm{min}^{-1}\right)$} & Pre-exercise & $10.3(1.0)$ & $12.8(3.0)^{* \S}$ & $9.5(0.9)$ \\
\hline & Exhaustion & $118.1(17.0)$ & $116.5(22.0)^{\S}$ & $75.3(11.1)^{*}$ \\
\hline & At $30 \mathrm{~min}$ post-exercise & $16.1(3.3)$ & $14.7(3.2)^{\S}$ & $11.2(1.7)^{*}$ \\
\hline \multirow{3}{*}{ Blood $\mathrm{pH}$} & Pre-exercise & $7.401(0.016)$ & $7.437(0.053)$ & $7.396(0.034)$ \\
\hline & Minimum value & $7.178(0.059)$ & $7.246(0.067)^{*}$ & $7.295(0.033)^{*}$ \\
\hline & At $30 \mathrm{~min}$ post-exercise & $7.337(0.038)$ & $7.407(0.029)^{*}$ & $7.381(0.027)^{*}$ \\
\hline \multirow[t]{3}{*}{ Blood $\mathrm{HCO}_{3}^{-}(\mathrm{mM})$} & Pre-exercise & $24.0(1.5)$ & $27.1(2.1)^{*}$ & $24.9(3.0)$ \\
\hline & Minimum value & $12.8(2.7)$ & $13.4(2.8)^{\S}$ & $18.4(1.4)^{*}$ \\
\hline & At $30 \mathrm{~min}$ post-exercise & $18.7(3.1)$ & $19.5(2.3)^{\S}$ & $23.1(2.5)^{*}$ \\
\hline \multirow[t]{3}{*}{ Base excess $(\mathrm{mEq} / \mathrm{l})$} & Pre-exercise & $0.2(1.3)$ & $3.3(0.8)^{*}$ & $0.6(2.1)$ \\
\hline & Minimum value & $-15.4(3.2)$ & $-12.8(4.0)^{\S}$ & $-7.5(2.1)^{*}$ \\
\hline & At $30 \mathrm{~min}$ post-exercise & $-5.6(3.3)$ & $-3.5(2.2)$ & $-1.2(2.5)^{*}$ \\
\hline
\end{tabular}

The online version of the original article can be found at http:// dx.doi.org/10.1007/s00421-003-0975-3

T. Kato $(\bowtie) \cdot$ Y. Matsumura $\cdot$ A. Tsukanaka $\cdot$ T. Harada

M. Kosaka · N. Matsui

Laboratory for Exercise Physiology and Biomechanics,

Chukyo University, 101 Tokodachi, Kaizu-cho Toyota,

470-0393 Aichi, Japan

E-mail: tkato@seiken.sass.chukyo-u.ac.jp

Tel.: + 81-565-461211

Fax: $+81-565-461272$ 